\title{
Retrospective analysis of KRAS status in metastatic colorectal cancer patients: a single-center feasibility study
}

This article was published in the following Dove Press journal:

Clinical and Experimental Gastroenterology

17 September 2012

Number of times this article has been viewed

\author{
Jonathan Montomoli' \\ Stephen Jacques \\ Hamilton-Dutoit ${ }^{2}$ \\ Trine Frøslev' \\ Aliki Taylor ${ }^{3}$ \\ Rune Erichsen' \\ 'Department of Clinical Epidemiology, \\ Aarhus University Hospital, Aarhus, \\ Denmark; ${ }^{2}$ Institute of Pathology, \\ Aarhus University Hospital, Aarhus, \\ Denmark; ${ }^{3}$ Centre for Observational \\ Research, Amgen, Uxbridge, UK
}

Background: The occurrence of $K R A S$ mutations and their association with prognosis in metastatic colorectal cancer patients is not well documented in population-based studies.

Objectives: To examine the feasibility of identifying archived colorectal cancer specimens, and through linkage with nationwide Danish population-based databases to investigate the prevalence of KRAS mutations and their association with colorectal cancer survival.

Methods: We used the Danish Pathology Database to identify the physical location of primary (or in some cases secondary) tumor specimens from selected metastatic colorectal cancer patients referred to our hospital for palliative chemotherapy between November 1, 2008 and September 30, 2009. Routinely stored paraffin tissue blocks were obtained from the pathology archives of the originating hospital. KRAS mutation tumor status was assessed for each patient using the commercialized TheraScreen KRAS Mutation Kit. Using the unique identifier number, we linked the patients to the Danish National Registry of Patients and the Danish Civil Registration System to obtain data on date of first colorectal cancer diagnosis and follow-up status. We estimated prevalence of KRAS mutations and the 1-, 2-, and 5-year survival after colorectal cancer diagnosis using the Kaplan-Meier technique.

Results: We identified 106 metastatic colorectal cancer patients (64\% males). All were successfully linked to the registries, and archived tumor-tissue samples were obtained and analyzed in each case. The overall prevalence of $K R A S$ mutations was 55\%, and 1-, 2-, and 5-year overall survival after colorectal cancer diagnosis was $91 \%, 68 \%$, and $25 \%$, respectively.

Conclusion: It is feasible to use Danish population-based registries to obtain archived tissue samples from metastatic colorectal cancer patients, and to estimate prevalence of KRAS mutation and subsequently evaluate the association with colorectal cancer survival.

Keywords: feasibility study, $K R A S$, colorectal neoplasms, survival, registries

\section{Introduction}

Colorectal cancer (CRC) is among the most common cancers in the Westernized world, and in Denmark more than 4000 new cases are diagnosed annually. ${ }^{1}$ Survival varies with cancer stage at diagnosis, thus the 5-year survival of metastatic CRC (mCRC) is $10 \%$, while that of early stage carcinoma is over $90 \%{ }^{2} \mathrm{CRC}$ with localized and regional spread benefits significantly from surgery and treatment with conventional chemotherapy. ${ }^{3}$ In contrast, treatment options for $\mathrm{mCRC}$ are still limited. Recent efforts to improve outcomes among mCRC patients have focused on the combination of standard chemotherapy with agents targeting biological pathways central to cancer pathogenesis. ${ }^{4,5}$ In particular, epidermal growth factor receptor (EGFR) inhibitors have been shown to improve overall and progression-free survival in these tumors. ${ }^{6-8}$
Correspondence: Jonathan Montomol Department of Clinical Epidemiology, Aarhus University Hospital,

Olof Palmes Allé 43-45,

DK-8200 Aarhus N, Denmark

Tel +4587 I 68252

Fax +4587167215

Email jm@dce.au.dk 
Importantly, these agents seem only to improve prognosis in mCRC patients with the wild-type guanosine triphosphatase $K R A S$ and not in patients with mCRC carrying KRAS mutations. ${ }^{6} K R A S$ is one of the most frequently activated oncogenes in several types of human cancer, and its mutations lead to constitutive activation of KRAS-signaling pathways, leading to increased and unregulated cellular proliferation and malignant transformation. ${ }^{9}$

While abundant data on KRAS mutations in mCRC have come from hospital-based studies and clinical trials, only a few studies have reported on KRAS mutations in mCRC occurring in population-based patient samples. ${ }^{10,11}$ Therefore, much is still to be investigated regarding the association between KRAS mutations and mCRC prognosis in the population-based setting. In view of this, we conducted a feasibility study to demonstrate ease of access to archived tissue samples from mCRC patients in Denmark, and the utility of a widely used commercial kit to test retrospectively for $K R A S$ status in such routinely processed tissue. Furthermore, our study underlines the potential of the present study design to be used for carrying out prognostic research by linking to population-based Danish registries.

\section{Materials and methods}

Widespread health registration occurs in Denmark under the auspices of the Danish National Health Service, which provides what is essentially a monopoly service of free (taxfunded) medical care for all Danish residents. Information can be efficiently linked through the unique ten-digit personal identifier (CPR number) assigned to each Danish citizen at birth or on immigration. ${ }^{12}$

\section{Specimens from colorectal cancer patients with metastatic disease}

The Danish National Pathology Registry (and its underlying national registration database, the Danish Pathology Data Bank) has a complete record of all pathology specimens examined in Denmark since January 1, 1997. The registry is updated daily, and records the unique ID number of the specimen and the examining pathology department, the date of examination, the CPR number of the patient, and the pathology diagnosis based on the Danish Systematized Nomenclature of Medicine codes. ${ }^{13-15}$ Using this registry, it is possible to identify all patients diagnosed with CRC (based on tumor tissue) nationwide. Furthermore, for each patient, it is possible to obtain a record of all pathology specimens obtained over time, the date of their removal, and the exact location of the archived samples.
For this feasibility study, we only included mCRC patients referred consecutively to our hospital for palliative chemotherapy during the study period (between November 1 , 2008 and September 30, 2009). We used this restricted approach to minimize costs for this small feasibility study. Using the local Danish Pathology Data Bank, we identified for each patient the primary diagnostic CRC tissue specimen and all subsequent primary and secondary CRC pathology specimens. For each sample, the date and place of removal and the physical location of the archived tissue specimens were available. Primary CRC tissue blocks (or if these were not sufficient for analysis, secondary tumor tissue blocks) were obtained from the pathology archive at the originating hospital. Submitting pathology departments were mostly from the western part of Denmark, as this is the area primarily served by our hospital as reference center, but also included some pathology departments from across the country.

\section{KRAS analysis}

$K R A S$ analyses were conducted at the Institute of Pathology, Aarhus University Hospital, using routinely processed and stored formalin-fixed paraffin-embedded CRC samples. Analyses were performed on primary tumors, or if there was insufficient primary tissue, on metastases. Both biopsy and resection specimens were included. Before DNA extraction, a pathologist confirmed the presence of adequate amounts of tumor tissue in each slide from the study paraffin blocks. If the percentage of tumor cells was below $10 \%$, tumor-cell enrichment was achieved by macrodissection of object-glass mounted, unstained sections. Using a routinely stained hematoxylin and eosin section as a guide, the major part of the nonneoplastic tissue areas was removed by scraping, before submitting the remaining tumor-rich areas for analysis.

For the KRAS analyses, we used the commercially available DxS TheraScreen KRAS Mutation Kit (Qiagen, Copenhagen, Denmark). This is the standard method used in our department for routine diagnostic $K R A S$-mutation detection. The test is widely used for site-specific analysis of the clinically most important $K R A S$ mutations. It is highly selective, sensitive, and robust, using allele-specific amplification combined with real-time polymerase chain reaction to detect seven key mutations in codons 12 and 13 of the KRAS gene (Gly12Asp [GGT > GAT], Gly12Ala [GGT > GCT], Gly12Val [GGT > GTT], Gly12Ser [GGT > AGT], Gly12Arg [GGT > CGT], Gly12Cys [GGT > TGT], and Gly13Asp [GGC > GAC]). By comparing control- and mutant-sample reactions, the assay can detect $1 \%$ of mutant DNA in a 
background of wild-type genomic DNA, provided that there is enough DNA of sufficient quality. ${ }^{16}$

\section{Linkage to other registries}

To test the feasibility of performing future prognostic studies, we linked our patients to the Danish National Registry of Patients. This registry covers a population of approximately 6 million in all Danish hospitals and contains information of all nonpsychiatric hospitalization since 1977 and outpatient contacts since $1995 .{ }^{17}$ Each record includes date of admission and discharge, surgical procedures carried out, and up to 20 discharge diagnoses coded by doctors according to the International Classification of Diseases (ICD), eighth revision, until the end of 1993, and according to the tenth revision thereafter. ${ }^{17}$ For this feasibility study, we collected the date of first diagnosis of CRC (ICD-10: C18-C20), the overall annual number of new CRCs diagnosed in Denmark for the period from which the specimens originated, and information about chemotherapy administered after first CRC diagnosis (cytostatic chemotherapy and/or treatment with cetuximab or other monoclonal antibodies). In addition, the registry could also be used to obtain information on, for example, comorbid diseases and surgical procedures.

We followed the patients from date of first CRC diagnosis until death, emigration, or January 1, 2011, whichever came first. Data on the overall number of Danish residents, death, or emigration were obtained by linkage to the Civil Registration System, which is updated daily and tracks vital status (dead or alive) and the residence of all Danish residents since April 1, 1968.

\section{Statistical analysis}

We estimated the prevalence of $K R A S$ mutant and wild type by dividing the number of patients with mutant and with wild-type $K R A S$ status by the total number of patients. Prevalence was estimated for all patients and stratified by gender. We estimated median, 1-, 2-, and 5-year survival and corresponding 95\% confidence intervals (CIs) after KRAS analysis using the Kaplan-Meier technique. We stratified survival estimates by gender and by KRAS status (wild type vs mutations [all seven mutations combined]).

\section{Results}

We identified $106 \mathrm{mCRC}$ (median age at CRC diagnosis: 61 years) patients; $64 \%$ were males. We were able to locate archived paraffin-embedded cancer tissues from all patients, and in each case to extract sufficient DNA for $K R A S$ analysis. Tissues used in this study originated from the period
1998-2009. However, the majority of tissue blocks were from the years 2005-2009, with only six (5.7\%) samples from before 2005. The underlying total number of new CRC diagnoses in Denmark in the 1998-2009 period was 46,596, reflecting an increase in the annual number of cases from 3645 in 1998 to 3921 in 2005 and 4289 in 2009. Furthermore, the cancer cases arose from a population that increased from 5.30 million in 1998 to 5.51 million in 2009 .

For all cases, successful linkage to other registries was obtained. Thirty-three (31.1\%) patients received only cytostatic treatment. The remaining $73(68.9 \%)$ patients were treated with cytostatic drugs in combination with cetuximab $(\mathrm{n}=22)$ or other monoclonal antibodies $(\mathrm{n}=51)$. Overall, the prevalence of KRAS mutations was $55 \%$ (Table 1 ). The most prevalent mutations were Gly12Val (15.1\%) and Gly12Asp (14.1\%).

One, 2, and 5 years after CRC diagnosis, the overall survival among mCRC patients was 91\% (95\% CI, 0.83-0.95), 68\% (95\% CI, 0.58-0.76), and 25\% (95\% CI, 0.16-0.35), respectively (Table 2). Median survival after CRC diagnosis among patients with wild-type and KRAS mutations was 3.7 years (95\% CI, 2.2-4.9) and 2.6 years (95\% CI, 2.0-3.4), respectively.

\section{Discussion}

In this feasibility study, we have outlined a retrospective study design that takes advantage of the nationwide Danish population-based databases and comprehensive archives of CRC specimens. This methodology seems appropriate for evaluating the prevalence and potential prognostic impact of $K R A S$ mutation in mCRC patients. The study confirms that it is feasible in a Danish population-based setting to obtain archived paraffin-embedded CRC tissue samples, utilize standardized $K R A S$-mutation analyses, and link to nationwide

Table I KRAS mutations in metastatic colorectal cancer patients included in this study

\begin{tabular}{|c|c|c|c|}
\hline & Female (\%) & Male (\%) & All (\%) \\
\hline Total number of patients & $38(35.9)$ & $68(64.2)$ & $106(100)$ \\
\hline \multicolumn{4}{|l|}{ KRAS } \\
\hline Wild type & $17(44.7)$ & $31(45.6)$ & $48(45.3)$ \\
\hline Mutations overall & $21(55.3)$ & $37(54.4)$ & $58(54.7)$ \\
\hline Gly12Asp & $2(5.3)$ & $13(19.1)$ & $15(14.1)$ \\
\hline Gly I2Ala & $3(7.9)$ & $3(4.4)$ & $6(5.7)$ \\
\hline Gly I2Val & $8(21.1)$ & $8(11.8)$ & $16(15.1)$ \\
\hline Gly12Ser & $2(5.3)$ & $\mathrm{I}(1.5)$ & $3(2.8)$ \\
\hline Gly|2Arg & $0(0.0)$ & $3(4.4)$ & $3(2.8)$ \\
\hline Gly 12Cys & $2(5.3)$ & $2(2.9)$ & $4(3.8)$ \\
\hline Gly13Asp & $2(5.3)$ & $6(8.8)$ & $8(7.6)$ \\
\hline Gly $12 \mathrm{Ala} / \mathrm{I} 2 \mathrm{Ser}$ & $2(5.3)$ & $\mathrm{I}(\mathrm{I} .5)$ & $3(2.8)$ \\
\hline
\end{tabular}


Table 2 Median, I-, 2-, and 5-year survival in metastatic colorectal cancer patients included in the study

\begin{tabular}{|c|c|c|c|c|}
\hline & \multicolumn{4}{|c|}{ Survival ( $95 \%$ confidence interval) } \\
\hline & Median survival, years & I year & 2 years & 5 years \\
\hline All $(n=106)$ & $2.88(2.15-3.63)$ & $0.91(0.83-0.95)$ & $0.68(0.57-0.78)$ & $0.25(0.16-0.35)$ \\
\hline Male $(n=68)$ & $3.44(2.14-4.07)$ & $0.90(0.80-0.95)$ & $0.66(0.53-0.76)$ & $0.29(0.17-0.4 I)$ \\
\hline Female $(n=38)$ & $2.55(2.05-2.95)$ & $0.92(0.78-0.97)$ & $0.7 \mathrm{I}(0.53-0.83)$ & $0.07(0.06-0.33)$ \\
\hline KRAS wild type $(\mathrm{n}=48)$ & $3.73(2.18-4.90)$ & $0.92(0.79-0.97)$ & $0.73(0.58-0.83)$ & $0.32(0.18-0.47)$ \\
\hline KRAS mutated $(\mathrm{n}=58)$ & $2.55(1.96-3.44)$ & $0.90(0.78-0.95)$ & $0.63(0.49-0.74)$ & $0.19(0.09-0.31)$ \\
\hline
\end{tabular}

registries to obtain information relevant for the study aim. We estimated that the prevalence of KRAS mutations was $55 \%$, and that these patients may have a slightly lower survival than those with $K R A S$ wild type.

Whereas the methodology of this study has proven its suitability for use in future studies, the reported KRAS prevalence and survival estimates need to be interpreted with caution for different reasons. Firstly, we included a relatively small nonrandom study sample, and therefore our results were imprecise and might not be generalizable to all mCRC patients. This may be the reason previous studies have reported substantially lower KRAS-mutation prevalence (approximately $20 \%-40 \%$ of CRC patients). ${ }^{18-22}$ Hence, in the Catalogue of Somatic Mutations in Cancer database, ${ }^{23}$ somatic missense KRAS mutations were reported in $35 \%$ of patients with colon adenocarcinoma. However, a previous study reported a $K R A S$-mutation prevalence in patients with CRC metastases of $48 \%$, which is closer to our findings, and even higher among CRC patients with lung and brain metastases. ${ }^{24}$ Secondly, since we followed patients from date of first CRC diagnosis, but only included those who lived long enough to be eligible for KRAS testing, we probably have overestimated survival as compared to the general mCRC patients (immortal time bias). Finally, previous studies used different criteria to identify the onset of follow-up, thus some considered the date of EGFRinhibitor treatment, ${ }^{25,26}$ while others used the date of other treatments ${ }^{22}$ or date of CRC diagnosis regardless of cancer stage. ${ }^{21}$ Nonetheless, a single-institution-based study reported better survival after CRC surgery in patients with at least one liver metastasis, comparing $K R A S$ wild type vs $K R A S$-mutated tumors. Hence, 2- and 4-year survivals were $74 \%$ and $51 \%$, respectively, in patients with $K R A S$ wild type and $60 \%$ and $20 \%$ in patients with $K R A S$-mutated cancers. $^{22}$ Although we included a relatively small sample, our survival estimates are broadly in accordance with those previously published, providing some validation to our methodology.
The methods used in our study could be elaborated and refined in potential larger studies, which would include a randomly selected sample of CRC patients to ensure generalizability. The use of a population-based design within a free tax-supported universal health-care system with complete hospital history and follow-up would reduce the risk of referral, diagnostic, and information bias. Furthermore, linkage to other databases such as the Danish Cancer Registry ${ }^{27}$ and the Danish Colorectal Cancer Group Database $^{28}$ could provide additional detailed information; both registries have been shown to be highly complete and valid. ${ }^{27,28}$ Thus, the Danish Cancer Registry has kept records of all incident malignant neoplasms in Denmark since 1943, and its data include, among other things, date of cancer diagnosis, tumor spread at diagnosis (localized, regional, metastasized, or unknown), and baseline treatments. ${ }^{27}$ The Danish Colorectal Cancer Group Database was founded in 1994. It collects information prospectively, including follow-up details on all patients with a first-time diagnosis of CRC at surgical departments in Denmark. This registry has the advantage of providing detailed information on a number of important factors, such as comorbidities, alcohol and tobacco consumption, diagnostics, and even follow-up treatments, and would therefore be a valuable source for future studies. ${ }^{28}$

Another factor to take into consideration in future studies is the choice of KRAS analysis. In this study, we used the TheraScreen KRAS Mutation Kit for detecting the seven key mutations. However, in future studies, additional mutations could be of importance, necessitating a different analytical approach. ${ }^{29}$ Moreover, further studies may investigate other biomarkers besides $K R A S$, such as mismatch-repair proteins and p53. ${ }^{19,30}$

In summary, this feasibility study has shown that it is possible to use nationwide Danish population-based registries to identify and obtain archived tissue samples from mCRC patients and subsequently to estimate the prevalence of $K R A S$ tumor mutation. A similar approach may be used in the future 
to facilitate studies of the association between $K R A S$ mutation and CRC prognosis.

\section{Disclosure}

This study received a research grant from Amgen.

\section{References}

1. Ferlay J, Parkin DM, Steliarova-Foucher E. Estimates of cancer incidence and mortality in Europe in 2008. Eur J Cancer. 2010;46(4):765-781.

2. Henry KA, Niu X, Boscoe FP. Geographic disparities in colorectal cancer survival. Int J Health Geogr. 2009;8:48.

3. Genovesi D, Cefaro GA, Vinciguerra A, et al. Retrospective long-term results and prognostic factors of postoperative treatment for UICC stages II and III rectal cancer. Tumori. 2009;95(6):675-682.

4. Cunningham D, Humblet Y, Siena S, et al. Cetuximab monotherapy and cetuximab plus irinotecan in irinotecan-refractory metastatic colorectal cancer. N Engl J Med. 2004;351(4):337-345.

5. Deschoolmeester V, Baay M, Specenier P, Lardon F, Vermorken JB. A review of the most promising biomarkers in colorectal cancer: one step closer to targeted therapy. Oncologist. 2010;15(7):699-731.

6. Dahabreh IJ, Terasawa T, Castaldi PJ, Trikalinos TA. Systematic review: anti-epidermal growth factor receptor treatment effect modification by KRAS mutations in advanced colorectal cancer. Ann Intern Med. 2011;154(1):37-49.

7. Jonker DJ, O'Callaghan CJ, Karapetis CS, et al. Cetuximab for the treatment of colorectal cancer. $N$ Engl J Med. 2007;357(20): 2040-2048.

8. Van Cutsem E, Peeters M, Siena S, et al. Open-label phase III trial of panitumumab plus best supportive care compared with best supportive care alone in patients with chemotherapy-refractory metastatic colorectal cancer. J Clin Oncol. 2007;25(13):1658-1664.

9. Kranenburg O. The KRAS oncogene: past, present, and future. Biochim Biophys Acta. 2005;1756(2):81-82.

10. Nosho K, Irahara N, Shima K, et al. Comprehensive biostatistical analysis of $\mathrm{CpG}$ island methylator phenotype in colorectal cancer using a large population-based sample. PLoS One. 2008;3(11):e3698.

11. Samowitz WS, Albertsen H, Herrick J, et al. Evaluation of a large, population-based sample supports a $\mathrm{CpG}$ island methylator phenotype in colon cancer. Gastroenterology. 2005;129(3):837-845.

12. Frank L. Epidemiology. When an entire country is a cohort. Science. 2000;287(5462):2398-2399.

13. College of American Pathologists. Systematized Nomenclature of Medicine. 2nd ed. Chicago: College of American Pathologists; 1979.

14. Vyberg M, Bjerregaard B, Bak M, Gram I, Hvolris H. Pathology database. Danish Society of Pathologic Anatomy and Cytology. Ugeskr Laeger. 2005;167(12-13):1401. Danish.

15. Erichsen R, Lash TL, Hamilton-Dutoit SJ, Bjerregaard B, Vyberg M, Pedersen L. Existing data sources for clinical epidemiology: the Danish National Pathology Registry and Data Bank. Clin Epidemiol. 2010, 2:51-56.
16. DxS-CancerMutation Products-Europe-TheraScreen K-RAS.Available from: http://www.qiagen.com/products/therascreenkrasrgqpcrkit. aspx\#Tabs $=t 1$. Accessed September 10, 2012.

17. Andersen TF, Madsen M, Jorgensen J, Mellemkjoer L, Olsen JH. The Danish National Hospital Register. A valuable source of data for modern health sciences. Dan Med Bull. 1999;46(3):263-268.

18. Hermsen M, Postma C, Baak J, et al. Colorectal adenoma to carcinoma progression follows multiple pathways of chromosomal instability. Gastroenterology. 2002;123(4):1109-1119.

19. Conlin A, Smith G, Carey FA, Wolf CR, Steele RJ. The prognostic significance of K-ras, p53, and APC mutations in colorectal carcinoma. Gut. 2005;54(9):1283-1286.

20. Knijn N, Mekenkamp LJ, Klomp M, et al. KRAS mutation analysis: a comparison between primary tumours and matched liver metastases in 305 colorectal cancer patients. Br J Cancer. 2011;104(6):1020-1026.

21. Deschoolmeester V, Boeckx C, Baay M, et al. KRAS mutation detection and prognostic potential in sporadic colorectal cancer using highresolution melting analysis. Br J Cancer. 2010;103(10):1627-1636.

22. Nash GM, Gimbel M, Shia J, et al. KRAS mutation correlates with accelerated metastatic progression in patients with colorectal liver metastases. Ann Surg Oncol. 2010;17(2):572-578.

23. Bamford S, Dawson E, Forbes S, et al. The COSMIC (Catalogue of Somatic Mutations in Cancer) database and website. Br J Cancer. 2004; 91(2):355-358.

24. Tie J, Lipton L, Desai J, et al. KRAS mutation is associated with lung metastasis in patients with curatively resected colorectal cancer. Clin Cancer Res. 2011;17(5):1122-1130.

25. De Roock W, Jonker DJ, Di Nicolantonio F, et al. Association of KRAS p.G13D mutation with outcome in patients with chemotherapyrefractory metastatic colorectal cancer treated with cetuximab. JAMA. 2010;304(16):1812-1820.

26. Bokemeyer C, Bondarenko I, Makhson A, et al. Fluorouracil, leucovorin, and oxaliplatin with and without cetuximab in the first-line treatmen of metastatic colorectal cancer. J Clin Oncol. 2009;27(5):663-671.

27. Storm HH, Michelsen EV, Clemmensen IH, Pihl J. The Danish Cancer Registry - history, content, quality and use. Dan Med Bull. 1997;44(5): 535-539.

28. Nickelsen TN, Harling H, Kronborg O, Bulow S, Jorgensen T. The completeness and quality of the Danish Colorectal Cancer clinical database on colorectal cancer. Ugeskr Laeger. 2004;166(36):3092-3095.

29. Smith G, Bounds R, Wolf H, Steele RJ, Carey FA, Wolf CR. Activating K-Ras mutations outwith 'hotspot' codons in sporadic colorectal tumours - implications for personalised cancer medicine. Br J Cancer. 2010;102(4):693-703

30. Jensen LH, Kuramochi H, Cruger DG, et al. Gene expression of the mismatch repair gene $\mathrm{MSH} 2$ in primary colorectal cancer. Tumour Biol. 2011;32(5):977-983.
Clinical and Experimental Gastroenterology

\section{Publish your work in this journal}

Clinical and Experimental Gastroenterology is an international, peerreviewed, open access journal, publishing all aspects of gastroenterology in the clinic and laboratory, including: Pathology, pathophysiology of gastrointestinal disease; Investigation and treatment of gastointestinal disease; Pharmacology of drugs used in the alimentary tract;

\section{Dovepress}

Immunology/genetics/genomics related to gastrointestinal disease. This journal is indexed on CAS. The manuscript management system is completely online and includes a very quick and fair peer-review system. Visit http://www.dovepress.com/testimonials.php to read real quotes from published authors. 